\title{
HotFlip: White-Box Adversarial Examples for Text Classification
}

\author{
Javid Ebrahimi*, Anyi Rao ${ }^{\dagger}$, Daniel Lowd*, Dejing Dou* \\ ${ }^{*}$ Computer and Information Science Department, University of Oregon, USA \\ \{javid, lowd, dou\}@cs.uoregon.edu \\ ${ }^{\dagger}$ School of Electronic Science and Engineering, Nanjing University, China \\ \{anyirao\}@smail.nju.edu.cn
}

\begin{abstract}
We propose an efficient method to generate white-box adversarial examples to trick a character-level neural classifier. We find that only a few manipulations are needed to greatly decrease the accuracy. Our method relies on an atomic flip operation, which swaps one token for another, based on the gradients of the onehot input vectors. Due to efficiency of our method, we can perform adversarial training which makes the model more robust to attacks at test time. With the use of a few semantics-preserving constraints, we demonstrate that HotFlip can be adapted to attack a word-level classifier as well.
\end{abstract}

\section{Introduction}

Adversarial examples are inputs to a predictive machine learning model that are maliciously designed to cause poor performance (Goodfellow et al., 2015). Adversarial examples expose regions of the input space where the model performs poorly, which can aid in understanding and improving the model. By using these examples as training data, adversarial training learns models that are more robust, and may even perform better on non-adversarial examples. Interest in understanding vulnerabilities of NLP systems is growing (Jia and Liang, 2017; Zhao et al., 2018; Belinkov and Bisk, 2018; Iyyer et al., 2018). Previous work has focused on heuristics for creating adversarial examples in the black-box setting, without any explicit knowledge of the model parameters. In the white-box setting, we use complete knowledge of the model to develop worst-case attacks, which can reveal much larger vulnerabilities.

We propose a white-box adversary against differentiable text classifiers. We find that only a few

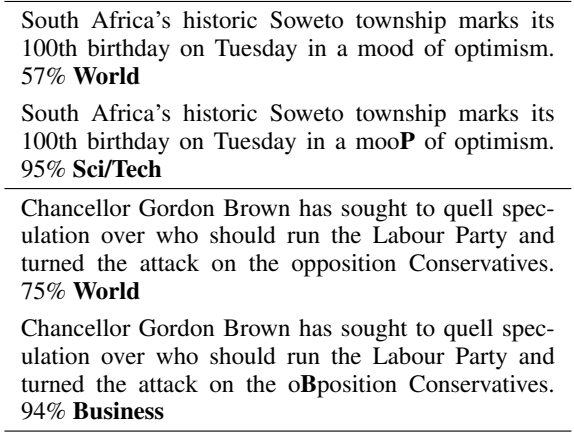

Table 1: Adversarial examples with a single character change, which will be misclassified by a neural classifier.

manipulations are needed to greatly increase the misclassification error. Furthermore, fast generation of adversarial examples allows feasible adversarial training, which helps the model defend against adversarial examples and improve accuracy on clean examples. At the core of our method lies an atomic flip operation, which changes one token to another by using the directional derivatives of the model with respect to the one-hot vector input.

Our contributions are as follows:

1. We propose an efficient gradient-based optimization method to manipulate discrete text structure at its one-hot representation.

2. We investigate the robustness of a classifier trained with adversarial examples, by studying its resilience to attacks and its accuracy on clean test data.

\section{Related Work}

Adversarial examples are powerful tools to investigate the vulnerabilities of a deep learning model (Szegedy et al., 2014). While this line of research has recently received a lot of attention in the deep learning community, it has a long history 
in machine learning, going back to adversarial attacks on linear spam classifiers (Dalvi et al., 2004; Lowd and Meek, 2005). Hosseini et al. (2017) show that simple modifications, such as adding spaces or dots between characters, can drastically change the toxicity score from Google's perspective API ${ }^{1}$. Belinkov and Bisk (2018) show that character-level machine translation systems are overly sensitive to random character manipulations, such as keyboard typos. They manipulate every word in a sentence with synthetic or natural noise. However, throughout our experiments, we care about the degree of distortion in a sentence, and look for stronger adversaries which can increase the loss within a limited budget. Instead of randomly perturbing text, we propose an efficient method, which can generate adversarial text using the gradients of the model with respect to the input.

Adversarial training interleaves training with generation of adversarial examples (Goodfellow et al., 2015). Concretely, after every iteration of training, adversarial examples are created and added to the mini-batches. A projected gradientbased approach to create adversarial examples by Madry et al. (2018) has proved to be one of the most effective defense mechanisms against adversarial attacks for image classification. Miyato et al. (2017) create adversarial examples by adding noise to word embeddings, without creating realworld textual adversarial examples. Our work is the first to propose an efficient method to generate real-world adversarial examples which can also be used for effective adversarial training.

\section{HotFlip}

HotFlip is a method for generating adversarial examples with character substitutions ("flips"). HotFlip also supports insertion and deletion operations by representing them as sequences of character substitutions. It uses the gradient with respect to a one-hot input representation to efficiently estimate which individual change has the highest estimated loss, and it uses a beam search to find a set of manipulations that work well together to confuse a classifier.

\subsection{Definitions}

We use $J(\mathbf{x}, \mathbf{y})$ to refer to the loss of the model on input $\mathbf{x}$ with true output $\mathbf{y}$. For example,

\footnotetext{
${ }^{1}$ https://www.perspectiveapi.com
}

for classification, the loss would be the log-loss over the output of the softmax unit. Let $V$ be the alphabet, $\mathrm{x}$ be a text of length $L$ characters, and $x_{i j} \in\{0,1\}^{|V|}$ denote a one-hot vector representing the $j$-th character of the $i$-th word. The character sequence can be represented by $\mathbf{x}=\left[\left(x_{11}, \ldots x_{1 n}\right) ; . .\left(x_{m 1}, \ldots x_{m n}\right)\right]$

wherein a semicolon denotes explicit segmentation between words. The number of words is denoted by $m$, and $n$ is the number of maximum characters allowed for a word.

\subsection{Derivatives of Operations}

We represent text operations as vectors in the input space and estimate the change in loss by directional derivatives with respect to these operations. Based on these derivatives, the adversary can choose the best loss-increasing direction. Our algorithm requires just one function evaluation (forward pass) and one gradient computation (backward pass) to estimate the best possible flip.

A flip of the $j$-th character of the $i$-th word $(a \rightarrow b)$ can be represented by this vector:

$$
\vec{v}_{i j b}=\left(\overrightarrow{0}, . . ;\left(\overrightarrow{0}, . .(0, . .-1,0, . ., 1,0)_{j}, . . \overrightarrow{0}\right)_{i} ; \overrightarrow{0}, . .\right)
$$

where -1 and 1 are in the corresponding positions for the $a$-th and $b$-th characters of the alphabet, respectively, and $x_{i j}^{(a)}=1$. A first-order approximation of change in loss can be obtained from a directional derivative along this vector:

$$
\nabla_{\vec{v}_{i j b}} J(\mathbf{x}, \mathbf{y})=\nabla_{x} J(\mathbf{x}, \mathbf{y})^{T} \cdot \vec{v}_{i j b}
$$

We choose the vector with biggest increase in loss:

$$
\max \nabla_{x} J(\mathbf{x}, \mathbf{y})^{T} \cdot \vec{v}_{i j b}=\max _{i j b}{\frac{\partial J}{\partial x_{i j}}}^{(b)}-{\frac{\partial J}{\partial x_{i j}}}^{(a)}
$$

Using the derivatives as a surrogate loss, we simply need to find the best change by calling the function mentioned in eq. 1, to estimate the best character change $(a \rightarrow b)$. This is in contrast to a naive loss-based approach, which has to query the classifier for every possible change to compute the exact loss induced by those changes. In other words, apart from the overhead of calling the function in eq. 1, one backward pass saves the adversary a large number of forward passes. 
Character insertion $^{2}$ at the $j$-th position of the $i$-th word can also be treated as a character flip, followed by more flips as characters are shifted to the right until the end of the word.

$$
\begin{gathered}
\max \nabla_{x} J(\mathbf{x}, \mathbf{y})^{T} \cdot \vec{v}_{i j b}=\max _{i j b}{\frac{\partial J}{\partial x_{i j}}}^{(b)}-{\frac{\partial J}{\partial x_{i j}}}^{(a)} \\
+\sum_{j^{\prime}=j+1}^{n}\left({\frac{\partial J}{\partial x_{i j^{\prime}}}}^{\left(b^{\prime}\right)}-{\frac{\partial J}{\partial x_{i j^{\prime}}}}^{\left(a^{\prime}\right)}\right)
\end{gathered}
$$

where $x_{i j^{\prime}}^{\left(a^{\prime}\right)}=1$ and $x_{i j^{\prime}-1}^{\left(b^{\prime}\right)}=1$. Similarly, character deletion can be written as a number of character flips as characters are shifted to the left. Since the magnitudes of direction vectors (operations) are different, we normalize by the $L_{2}$ norm of the vector i.e., $\frac{\vec{v}}{\sqrt{2 N}}$, where $\mathrm{N}$ is the number of total flips.

\subsection{Multiple Changes}

We explained how to estimate the best single change in text to get the maximum increase in loss. A greedy or beam search of $r$ steps will give us an adversarial example with a maximum of $r$ flips, or more concretely an adversarial example within an $L_{0}$ distance of $r$ from the original example. Our beam search requires only $\mathcal{O}(b r)$ forward passes and an equal number of backward passes, with $r$ being the budget and $b$, the beam width. We elaborate on this with an example: Consider the loss function $J($.$) , input x_{0}$, and an individual change $c_{j}$. We estimate the score for the change as $\frac{\partial J\left(x_{0}\right)}{\partial c_{j}}$. For a sequence of 3 changes $\left[c_{1}, c_{2}, c_{3}\right]$, we evaluate the "score" as follows.

$\operatorname{score}\left(\left[c_{1}, c_{2}, c_{3}\right]\right)=\frac{\partial J\left(x_{0}\right)}{\partial c_{1}}+\frac{\partial J\left(x_{1}\right)}{\partial c_{2}}+\frac{\partial J\left(x_{2}\right)}{\partial c_{3}}$

where $x_{1}$ and $x_{2}$ are the modified input after applying $\left[c_{1}\right]$ and $\left[c_{1}, c_{2}\right]$ respectively. We need $b$ forward and backward passes to compute derivatives at each step of the path, leading to $\mathcal{O}(b r)$ queries. In contrast, a naive loss-based approach requires computing the exact loss for every possible change at every stage of the beam search, leading to $\mathcal{O}(b r L|V|)$ queries.

\section{Experiments}

In principle, HotFlip could be applied to any differentiable character-based classifier. Here, we focus on the CharCNN-LSTM architecture (Kim

\footnotetext{
${ }^{2}$ For ease in exposition, we assume that the word size is at most $n-1$, leaving at least one position of padding at the end.
}

et al., 2016), which can be adapted for classification via a single dense layer after the last recurrent hidden unit. We use the AG's news dataset ${ }^{3}$, which consists of 120,000 training and 7,600 test instances from four equal-sized classes: World, Sports, Business, and Science/Technology. The architecture consists of a 2-layer stacked LSTM with 500 hidden units, a character embedding size of 25, and 1000 kernels of width 6 for temporal convolutions. This classifier was able to outperform (Conneau et al., 2017), which has achieved the state-of-the-art result on some benchmarks, on AG's news. The model is trained with SGD and gradient clipping, and the batch size was set to 64 . We used $10 \%$ of the training data as the development set, and trained for a maximum of 25 epochs. We only allow character changes if the new word does not exist in the vocabulary, to avoid changes that are more likely to change the meaning of text. The adversary uses a beam size of 10 , and has a budget of maximum of $10 \%$ of characters in the document. In Figure 1, we plot the success rate of the adversary against an acceptable confidence score for the misclassification. That is, we consider the adversary successful only if the classifier misclassifies the instance with a given confidence score. For this experiment, we create adversarial examples for $10 \%$ of the test set.

We compare with a (greedy) black-box adversary, which does not have access to model parameters, and simply queries the classifier with random character changes. Belinkov and Bisk (2018) define an attack, Key, in which a character is replaced with an adjacent character in the keyboard. We allow a stronger black-box attacker to change a character to any character in the alphabet, and we call it Key* ${ }^{*}$. As expected a white-box adversary is much more damaging, and has a higher success rate. As can be seen, the beam-search strategy is very effective in fooling the classifier even with an 0.9 confidence constraint, tricking the classifier for more than $90 \%$ of the instances. A greedy search is less effective especially in producing high-confidence scores. We use a maximum of $10 \%$ of characters in the document as the budget for the adversary, but our adversary changes an average of $4.18 \%$ of the characters to trick the classifier at confidence 0.5 . The adversary picks the flip operation around $80 \%$ of the times, and favors delete over insert by two to one.

\footnotetext{
${ }^{3}$ https://www.di.unipi.it/ gulli/
} 


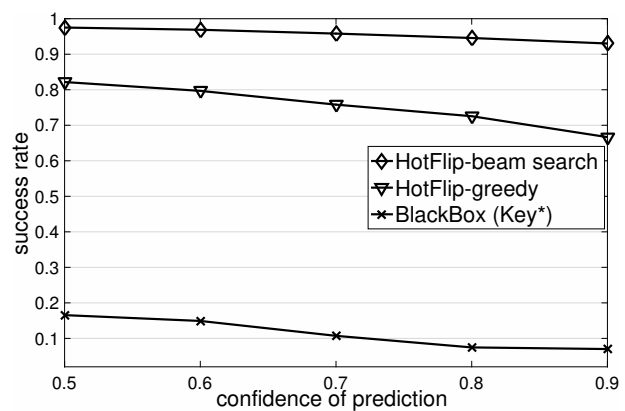

Figure 1: Adversary's success as a function of confidence.

\subsection{Robustness}

For our adversarial training, we use only use the flip operation, and evaluate models' robustness to this operation only. This is because insert and delete manipulations are $n$ times slower to generate, where $n$ is the number of maximum characters allowed for a word. For these experiments, we have no constraint on confidence score. We flip $r$ characters for each training sample, which was set to $20 \%$ of the characters in text after tuning, based on the accuracy on the development set. In addition, for faster generation of adversarial examples, we directly apply the top $r$ flips after the first backward pass, simultaneously ${ }^{4}$.

We use the full test set for this experiment, and we compare HotFlip adversarial training with the white-box (supervised) adversarial training (Miyato et al., 2017) that perturbs word embeddings, which we adapt to work with character embeddings. Specifically, the adversarial noise per character is constrained by the Frobenius norm of the embedding matrix composed of the sequence of characters in the word. We also create another baseline where instead of white-box adversarial examples, we add black-box adversarial examples $\left(\mathrm{Key}^{*}\right)$ to the mini-batches. As shown in Table 2 , our approach decreases misclassification error and dramatically decreases the adversary's success rate. In particular, adversarial training on real adversarial examples generated by HotFlip, is more effective than training on pseudo-adversarial examples created by adding noise to the embeddings.

The current error of our adversarially trained model is still beyond an acceptable rate; this is mainly because the adversary that we use at test time, which uses beam search, is strictly stronger than our model's internal adversary. This has been observed in computer vision where strongest ad-

\footnotetext{
${ }^{4}$ The adversary at test time would still use beam search.
}

\begin{tabular}{c|c|c} 
Method & Misc. error & Success rate \\
\hline Baseline & $8.27 \%$ & $98.16 \%$ \\
\hline Adv-tr (Miyato et al., 2017) & $8.03 \%$ & $87.43 \%$ \\
\hline Adv-tr (black-box) & $8.60 \%$ & $95.63 \%$ \\
\hline Adv-tr (white-box) & $\mathbf{7 . 6 5} \%$ & $\mathbf{6 9 . 3 2} \%$
\end{tabular}

Table 2: Comparison based on misclassification error on clean data and adversary's success rate.

versaries are not efficient enough for adversarial training, but can break models trained with weaker adversaries (Carlini and Wagner, 2017).

\subsection{Human Perception}

Our human evaluation experiment shows that our character-based adversarial examples rarely alter the meaning of a sentence. We conduct an experiment of annotating 600 randomly-picked instances annotated by at least three crowd workers in Amazon Mechanical Turk. This set contains 150 examples of each class of AG's-news dataset, all of which are correctly classified by the classifier. We manipulate half of this set by our algorithm, which can successfully trick the classifier to misclassify these 300 adversarial examples. The median accuracy of our participants decreased by $1.78 \%$ from $87.49 \%$ on clean examples to $85.71 \%$ on adversarial examples. Similar small drops in human performance have been reported for image classification (Papernot et al., 2016) and text comprehension (Jia and Liang, 2017).

\section{HotFlip at Word-Level}

HotFlip can naturally be adapted to generate adversarial examples for word-level models, by computing derivatives with respect to one-hot word vectors. After a few character changes, the meaning of the text is very likely to be preserved or inferred by the reader (Rawlinson, 1976), which was also confirmed by our human subjects study. By contrast, word-level adversarial manipulations are much more likely to change the meaning of text, which makes the use of semantics-preserving constraints necessary. For example, changing the word good to bad changes the sentiment of the sentence "this was a good movie". In fact, we expect the model to predict a different label after such a change.

To showcase the applicability of HotFlip to a word-level classifier, we use Kim's CNN (2014) trained for binary sentiment classification on the SST dataset (Socher et al., 2013). In order to create adversarial examples, we add constraints so that 


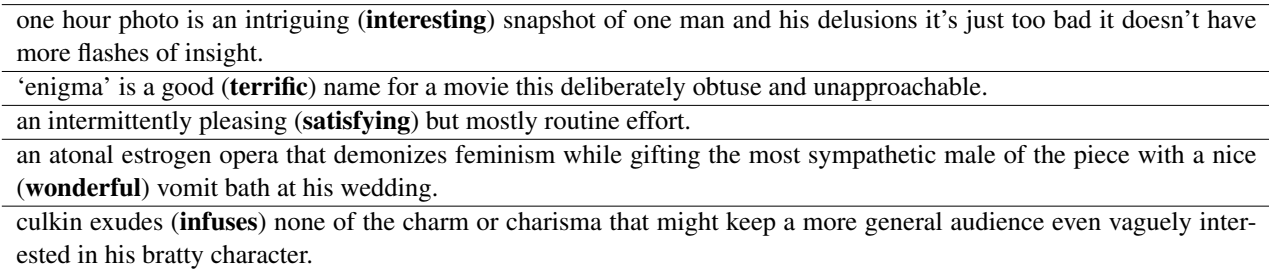

Table 3: Adversarial examples for sentiment classification. The bold words replace the words before them.

\begin{tabular}{l|l|l|l|l|l|l|l} 
past $\rightarrow$ pas!t & Alps $\rightarrow$ llps & talk $\rightarrow$ taln & local $\rightarrow$ loral & you $\rightarrow$ yoTu & ships $\rightarrow$ hips & actor $\rightarrow$ actr & lowered $\rightarrow$ owered \\
\hline pasturing & lips & tall & moral & Tutu & dips & act & powered \\
pasture & laps & tale & Moral & Hutu & hops & acting & empowered \\
pastor & legs & tales & coral & Turku & lips & actress & owed \\
Task & slips & talent & morals & Futurum & hits & acts & overpowered
\end{tabular}

Table 4: Nearest neighbor words (based on cosine similarity) of word representations from CharCNN-LSTM, picked at the output of the highway layers. A single adversarial change in the word often results in a big change in the embedding, which would make the word more similar to other words, rather than to the original word.

the resulting sentence is likely to preserve the original meaning; we only flip a word $w_{i}$ to $w_{j}$ only if these constraints are satisfied:

1. The cosine similarity between the embedding of words is bigger than a threshold (0.8).

2. The two words have the same part-of-speech.

3. We disallow replacing of stop-words, as for many of the stop-words, it is difficult to find cases where replacing them will still render the sentence grammatically correct. We also disallow changing a word to another word with the same lexeme for the same purpose.

Table 3 shows a few adversarial examples with only one word flip. In the second and the fourth examples, the adversary flips a positive word (i.e., good, nice) with highly positive words (i.e., terrific, wonderful) in an overall very negative review. These examples, albeit interesting and intuitive, are not abundant, and thus pose less threat to an NLP word-level model. Specifically, given the strict set of constraints, we were able to create only 41 examples ( $2 \%$ of the correctly-classified instances of the SST test set) with one or two flips.

For a qualitative analysis of relative brittleness of character-level models, we study the change in word embedding as an adversarial flip, insert, or delete operation occurs in Table 4 . We use the output of the highway layer as the word representation, and report the embedding for a few adversarial words, for which the original word is not among their top 5 nearest neighbors.

In a character-level model, the lookup operation to pick a word from the vocabulary is replaced by a character-sequence feature extractor which gives an embedding for any input, including OOV words which would be mapped to an UNK token in a word-level model. This makes the embedding space induced in character-level representation more dense, which makes characterlevel models more likely to misbehave under small adversarial perturbations.

\section{Conclusion and Future Work}

White-box attacks are among the most serious forms of attacks an adversary can inflict on a machine learning model. We create white-box adversarial examples by computing derivatives with respect to a few character-edit operations (i.e., flip, insert, delete), which can be used in a beam-search optimization. While character-edit operations have little impact on human understanding, we found that character-level models are highly sensitive to adversarial perturbations. Employing these adversarial examples in adversarial training renders the models more robust to such attacks, as well as more robust to unseen clean data.

Contrasting and evaluating robustness of different character-level models for different tasks is an important future direction for adversarial NLP. In addition, the discrete nature of text makes it a more challenging task to understand the landscape of adversarial examples. Research in this direction can shed light on vulnerabilities of NLP models.

\section{Acknowledgement}

This work was funded by ARO grant W911NF15-1-0265. 


\section{References}

Yonatan Belinkov and Yonatan Bisk. 2018. Synthetic and natural noise both break neural machine translation. In Proceedings of ICLR.

Nicholas Carlini and David Wagner. 2017. Towards evaluating the robustness of neural networks. In $\mathrm{Se}$ curity and Privacy (SP), 2017 IEEE Symposium on, pages 39-57. IEEE.

Alexis Conneau, Holger Schwenk, Loïc Barrault, and Yann Lecun. 2017. Very deep convolutional networks for text classification. In Proceedings of $E A C L$, volume 1, pages 1107-1116.

Nilesh Dalvi, Pedro Domingos, Sumit Sanghai, Deepak Verma, et al. 2004. Adversarial classification. In Proceedings of KDD, pages 99-108.

Ian J Goodfellow, Jonathon Shlens, and Christian Szegedy. 2015. Explaining and harnessing adversarial examples. In Proceedings of ICLR.

Hossein Hosseini, Sreeram Kannan, Baosen Zhang, and Radha Poovendran. 2017. Deceiving google's perspective api built for detecting toxic comments. arXiv preprint arXiv:1702.08138.

Mohit Iyyer, John Wieting, Kevin Gimpel, and Luke Zettlemoyer. 2018. Adversarial example generation with syntactically controlled paraphrase networks. arXiv preprint arXiv:1804.06059.

Robin Jia and Percy Liang. 2017. Adversarial examples for evaluating reading comprehension systems. In Proceedings of EMNLP.

Yoon Kim. 2014. Convolutional neural networks for sentence classification. In Proceedings of EMNLP.

Yoon Kim, Yacine Jernite, David Sontag, and Alexander M Rush. 2016. Character-aware neural language models. Proceedings of AAAI.

Daniel Lowd and Christopher Meek. 2005. Adversarial learning. In Proceedings of KDD, pages 641-647.

Aleksander Madry, Aleksandar Makelov, Ludwig Schmidt, Dimitris Tsipras, and Adrian Vladu. 2018. Towards deep learning models resistant to adversarial attacks. In Proceedings of ICLR.

Takeru Miyato, Andrew M Dai, and Ian Goodfellow. 2017. Adversarial training methods for semisupervised text classification. In Proceedings of ICLR.

Nicolas Papernot, Patrick McDaniel, Somesh Jha, Matt Fredrikson, Z Berkay Celik, and Ananthram Swami. 2016. The limitations of deep learning in adversarial settings. In Security and Privacy (EuroS\&P), 2016 IEEE European Symposium on, pages 372387. IEEE.

Graham Ernest Rawlinson. 1976. The Significance of Letter Position in Word Recognition. Ph.D. thesis, University of Nottingham.
Richard Socher, Alex Perelygin, Jean Wu, Jason Chuang, Christopher D Manning, Andrew Ng, and Christopher Potts. 2013. Recursive deep models for semantic compositionality over a sentiment treebank. In Proceedings of EMNLP, pages 1631-1642.

Christian Szegedy, Wojciech Zaremba, Ilya Sutskever, Joan Bruna, Dumitru Erhan, Ian Goodfellow, and Rob Fergus. 2014. Intriguing properties of neural networks. In Proceedings of ICLR.

Zhengli Zhao, Dheeru Dua, and Sameer Singh. 2018. Generating natural adversarial examples. In Proceedings of ICLR. 